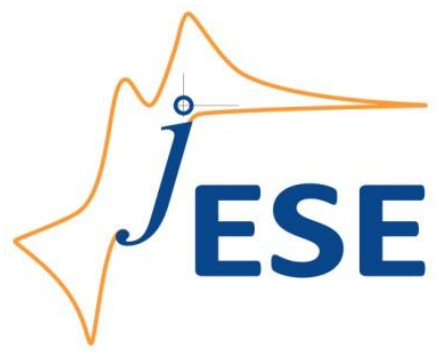

Open Access : : ISSN 1847-9286

www.jESE-online.org

Original scientific paper

\title{
A multichannel frequency response analyser for impedance spectroscopy on power sources
}

\author{
QUENTIN MEYER, SIMON BARASS, OLIVER CURNICK*, TOBIAS REISCH*, \\ DANIEL J. L. BRETT $\bowtie$
}

Electrochemical Innovation Laboratory, Centre for $\mathrm{CO}_{2}$ Technology, Department of Chemical Engineering, University College London, UK

*Intelligent Energy Ltd, Loughborough, Leicestershire, UK

${ }^{\square}$ Corresponding Author: E-mail: d.brett@ucl.ac.uk; Tel.: +44(0)207 679 3310; Fax: +44(0)207 3832348

Received: December 19, 2012; Published: June 12, 2013

\begin{abstract}
A low-cost multi-channel frequency response analyser (FRA) has been developed based on a DAQ (data acquisition)/LabVIEW interface. The system has been tested for electric and electrochemical impedance measurements. This novel association of hardware and software demonstrated performance comparable to a commercial potentiostat / FRA for passive electric circuits. The software has multichannel capabilities with minimal phase shift for 5 channels when operated below $3 \mathrm{kHz}$. When applied in active (galvanostatic) mode in conjunction with a commercial electronic load (by discharging a lead acid battery at 1.5 A) the performance was fit for purpose, providing electrochemical information to characterize the performance of the power source.
\end{abstract}

\section{Keywords}

LabVIEW, multi-channel impedance, electrical circuit, fuel cell, lead acid battery.

\section{Introduction}

Electrochemical impedance spectroscopy (EIS) is a powerful diagnostic technique that has been the subject of significant technical development over the last fifty years [1-3]. It has proven to be particularly useful in the fields of electrochemistry, corrosion [4], primary and secondary batteries [5,6] and fuel cells [7-9]. Advances in electronics and reduction in the cost of hardware has made the technique increasingly popular. However, EIS is still mainly used as a high-end lab-based diagnostic that requires relatively expensive hardware. This has hindered its uptake as a diagnostic tool used in routine industrial analysis or for on-line measurements of electrochemical power systems. For applications that would benefit from multichannel input (on different cells in a fuel cell stack, for example) the cost and complexity multiplies. Therefore, there is a need for a lowcost, modular approach for electrochemical impedance spectroscopy analysis. 
Alternative methods for performing multichannel EIS analysis have been attempted previously using Matlab GUI Builder [10] and a built-in 10 channels frequency analyser. Single channel EIS using commercially available DAQ (data acquisition) hardware and a LabVIEW interface has been attempted for fuel cell applications [11], but multichannel metrology has yet to be reported.

This paper details the development of UCL-FRA, a low-cost EIS system based on the use of a commercially available DAQ interface and LabVIEW software (DAQ/LabVIEW interface). Issues relating to the successful application of the technique are discussed, results of its operation in galvanostatic mode applied to a battery are presented and operation in multi-channel mode described.

\section{Experimental}

\section{Software}

The software used for data collection and processing was developed using LabVIEW 2011 (National Instruments) and was based on the use of VIs (virtual instruments) in the Sound and Vibration Toolkit library for generating and analyzing AC waveforms. The coding methodology required special consideration to account for low frequency detection, simultaneous sampling and multichannel responses. The multichannel response was achieved by outputting single discrete sine waves sequentially across the frequency range of choice, and measuring on 5 response channels simultaneously. The software is currently capable of 5 response channels, but can be modified to a higher number of channels to suit the application, provided that the performance of the hardware is adequate to ensure robust data collection. The processed signals are displayed as Nyquist and Bode plots in real time as the data is collected; the time for the software to process the signal does not impact on the rate at which the measurement can be made. An entire measurement from $10 \mathrm{kHz}$ to $0.1 \mathrm{~Hz}$ with 10 points per decade, takes about $400 \mathrm{~s}$, which is comparable to other commercial devices.

The signal sampling frequency is limited by the hardware used. High frequency sampling improves the accuracy of the measurement when analyzing high frequency signals. However, when sampling low frequencies, where the minimum sampling time is the inverse of the signal frequency, the data buffer becomes saturated. It is also necessary to respect the Nyquist-Shannon principle for robust data sampling [12]; e.g. for a maximum bandwidth of $10 \mathrm{kHz}$, the sampling rate cannot be smaller than $20 \mathrm{kS} / \mathrm{s}$. In practice, to avoid aliasing, a clock speed 10 times greater than the maximum speed of the measurement was used for each channel $(100 \mathrm{kS} / \mathrm{s})$. Therefore, the DAQ hardware suitable for this application needs at least $500 \mathrm{kS} / \mathrm{s}$, for 5 response channels.

\section{Hardware}

A USB data acquisition card (USB 6363, National Instrument) was used for data collection and signal generation. The DAQ has 16 bit resolution, with 32 input channels (16 differential), 4 analog outputs, a sampling rate of $1 \mathrm{MS} / \mathrm{s}$ and an output rate of $2.86 \mathrm{MS} / \mathrm{s}$. It was used in the $\pm 5 \mathrm{~V}$ range (or lower), with a resolution of $153 \mu \mathrm{V}$ (i.e. $10 \mathrm{~V} / 2^{16}$ ). All the response signals were recorded in differential mode.

The AC perturbation was generated as a single sine wave, switching between discrete levels from high to low frequency. The $A C+D C$ stimulus and response signals are both recorded by the DAQ and processed using a Fast Fourier Transform (FFT) to convert the signals from the time domain to the frequency domain.

Figure 1 shows the set-up used in this work. Electrical impedance measurements on 'dummy cells' were performed in potentiostatic mode, where the stimulus voltage is imposed to the 
system and the current response is recorded using the $22 \Omega$ series resistor as a shunt resistor. The electrochemical impedance measurements on the battery were performed in galvanostatic mode; the stimulus current is controlled using an analogue control port on the load unit and recorded using a current transducer.

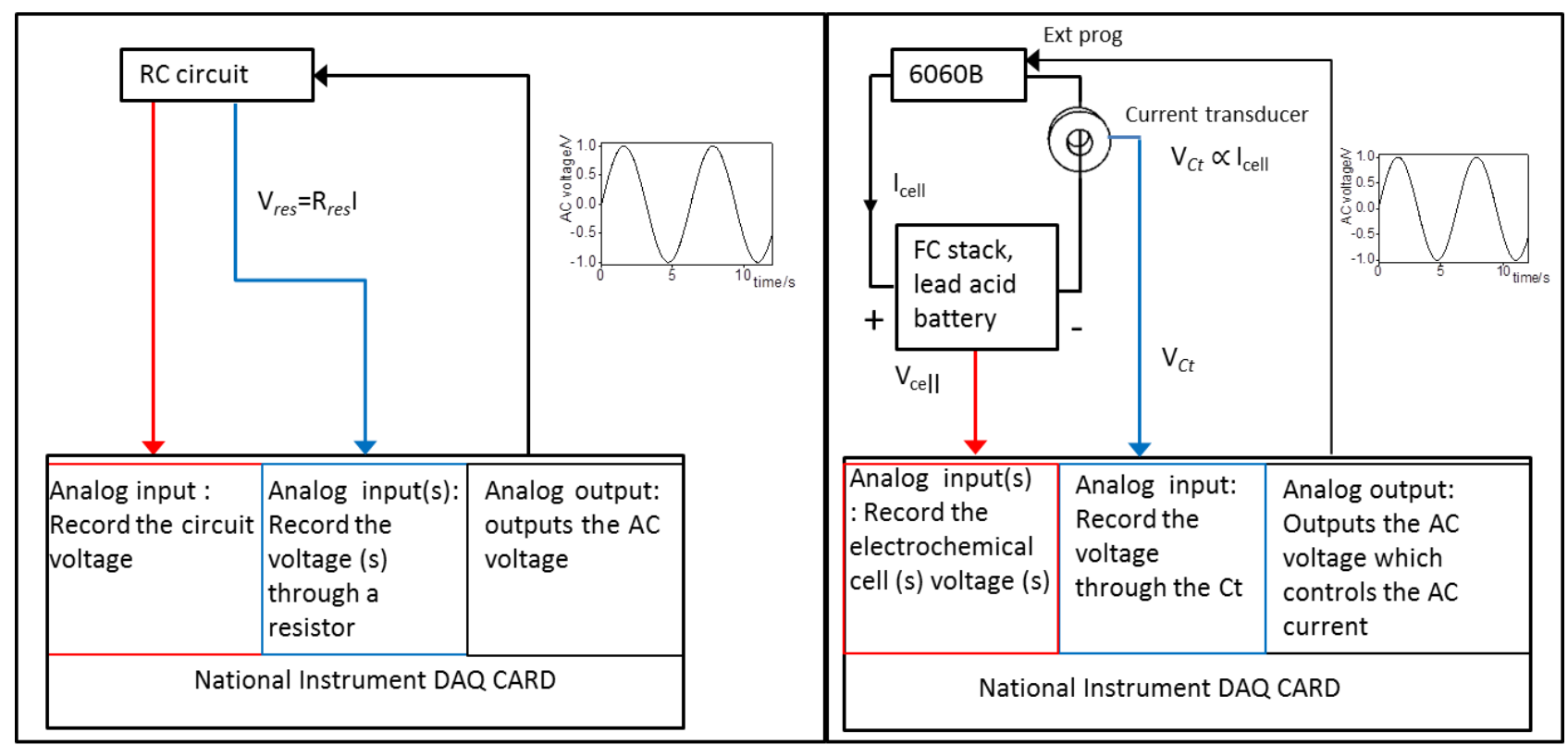

(a)

(b)

Figure 1. Electrical connection schematic for (a) electrical and (b) electrochemical impedance spectroscopy. Black line represents the voltage stimulus from the DAQ; blue the voltage as a proxy for the current and red the actual voltage from the cell.

\section{Measurement procedure for passive components in potentiostatic mode}

An electric circuit (dummy cell) was used for electrical impedance testing. This was composed of a $22 \Omega$ resistor $R_{1}$ in series with an RC parallel combination (4.7 $\mu \mathrm{F}$ capacitor $C_{1}$ and $200 \Omega$ resistor $R_{2}$ ). This circuit was connected to the DAQ card to enable single and multiple channel response. The series resistor $R_{1}$ is used as a shunt to derive the current through the circuit.
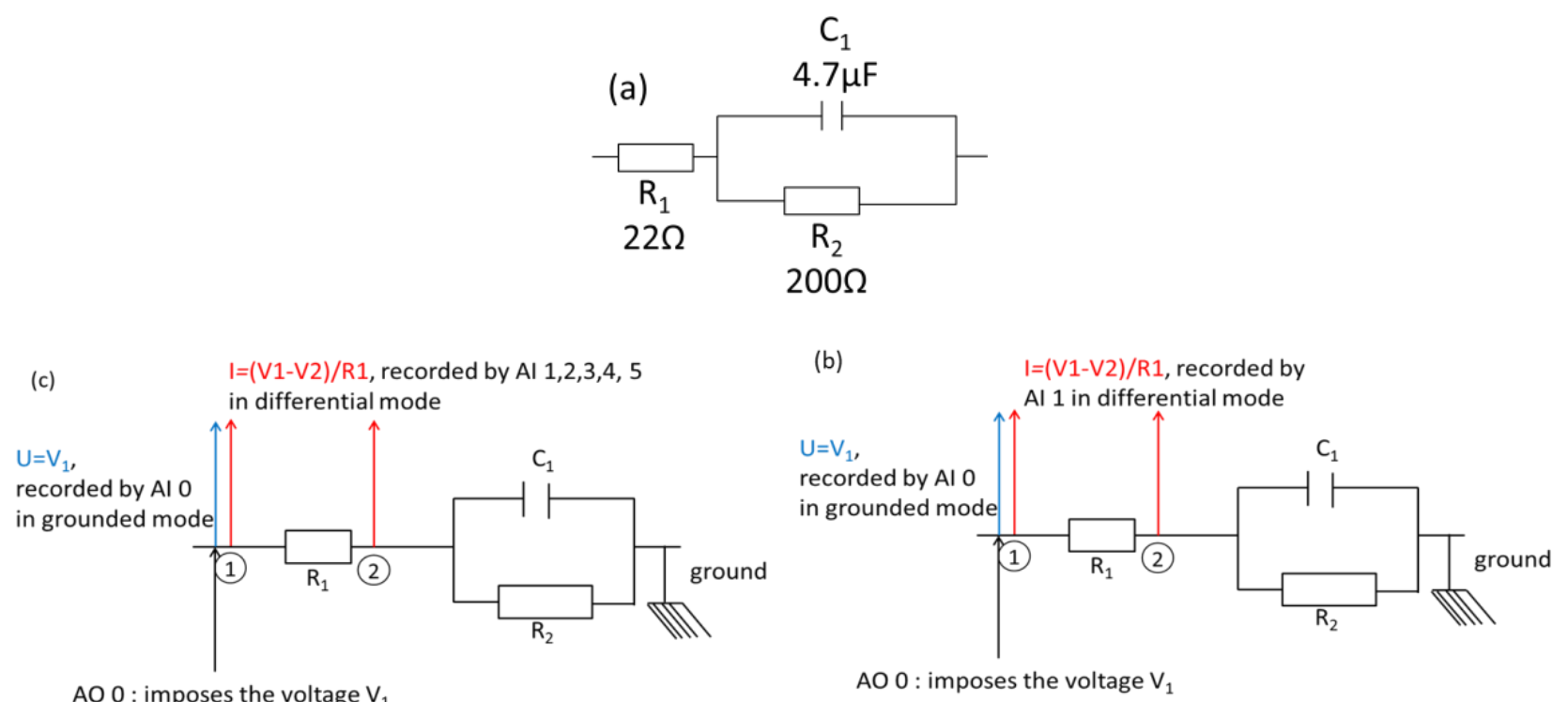

AO 0 : imposes the voltage $V_{1}$

$\mathrm{AO} 0$ : imposes the voltage $\mathrm{V}_{1}$

Figure 2. (a) Electrical circuit comprising the 'dummy cell'; (b) connections for measuring voltage across the shunt resistor in single and (c) multiple-input mode. AO: analog output. Al: analog input. 
Measurement procedure for electrochemical impedance in galvanostatic mode

In order to trial the system on an electrochemical power source, a commercially available lead acid battery ( $6 \mathrm{~V}, 10 \mathrm{Ah}$, Yuasa) was tested. An electronic load (6060B Agilent) with a bandwidth of $10 \mathrm{kHz}$ was used to control discharge rate. The load is equipped with an analogue input capable of remotely controlling the current that is passed [13], a $0-10 \mathrm{~V}$ signal from the DAQ can be converted into a 0-60 A current by the load unit. A current transducer, with a frequency bandwidth of $150 \mathrm{kHz}$ (ITS-ULTRASTAB-60, LEM) capable of zero flux detection was used to record the $A C$ and $D C$ current, and convert it to a voltage across a resistor. The voltage of the battery and the voltage across the burden resistor are then recorded at the same time; consequently the transfer function can be extracted, as described in Figure 1(b). The load unit has a bandwidth of 10 $\mathrm{kHz}$, and therefore some attenuation could be expected for frequencies higher than $2 \mathrm{kHz}$. For comparison with a commercially available high current potentiostat an IviumSTAT A11700 (10 V, 10 A, Ivium, Netherlands) was used. For both systems the AC perturbation was equivalent to $75 \mathrm{~mA}$ imposed across a frequency range of $10 \mathrm{kHz}-1 \mathrm{~Hz}$.

\section{Results and Discussion}

Assessment of DAQ hardware as a function generation and frequency response analyser.

It is important to ensure that there are no artifacts or significant phase shifts introduced by the hardware/software combination. Figure 3(a) shows that when the input and output of the DAQ are connected together there is agreement between the two in terms of the frequency generated and measured. Figure 3(b) shows a Bode plot of magnitude and phase. It can be seen that the magnitude of the signal is constant across the range and that there is only a small phase shift introduced above $1 \mathrm{kHz}$ (<0.03 degrees at frequencies up to $10 \mathrm{kHz}$ ).
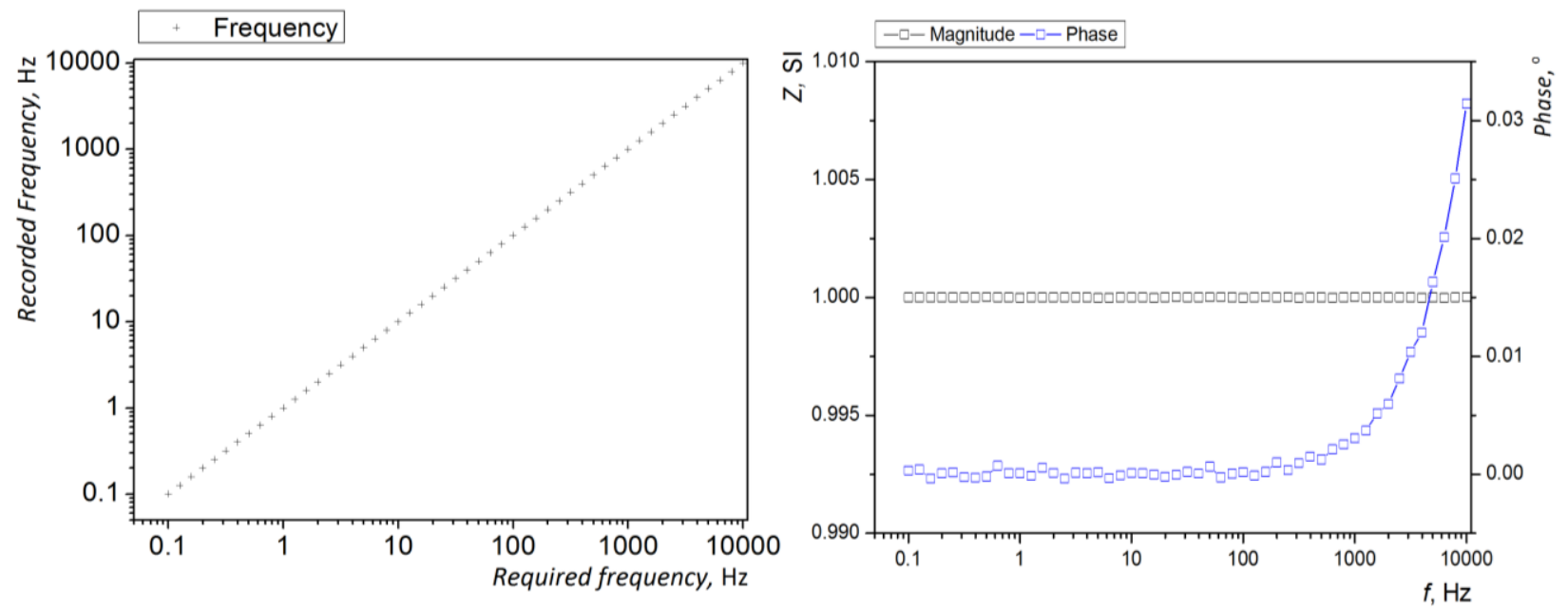

Figure 3. (a) Frequency generated versus recorded frequency sweep, from $10 \mathrm{kHz}$ to $0.5 \mathrm{~Hz}$.

(b) Phase and Magnitude for the impedance between two channels recording the same signal.

\section{Electrical impedance: single channel mode}

The EIS system was tested in single channel mode on the circuit shown in Figure 2(b). Figure 4 shows Nyquist and Bode plots comparing the response obtained from the UCL system, the commercial (Ivium) frequency response analyzer and the model simulation based on the components used in the circuit. It can be seen that excellent agreement is obtained between each, over the entire frequency range $(10 \mathrm{kHz}$ to $0.1 \mathrm{~Hz})$. This demonstrates that the system is suitable for performing EIS on passive (non power source) systems. 


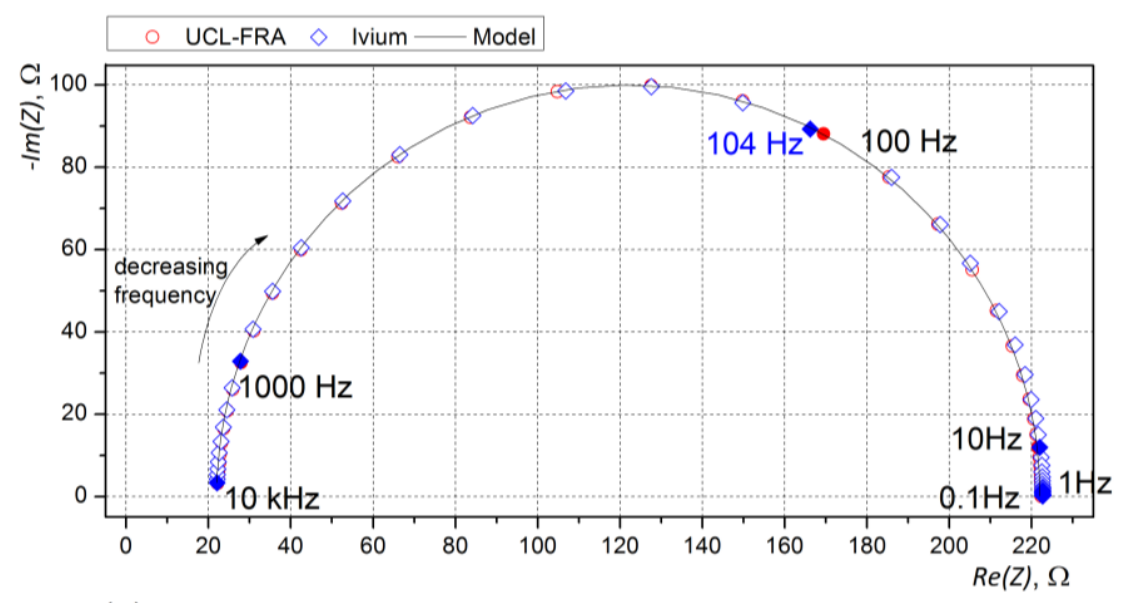

(a)
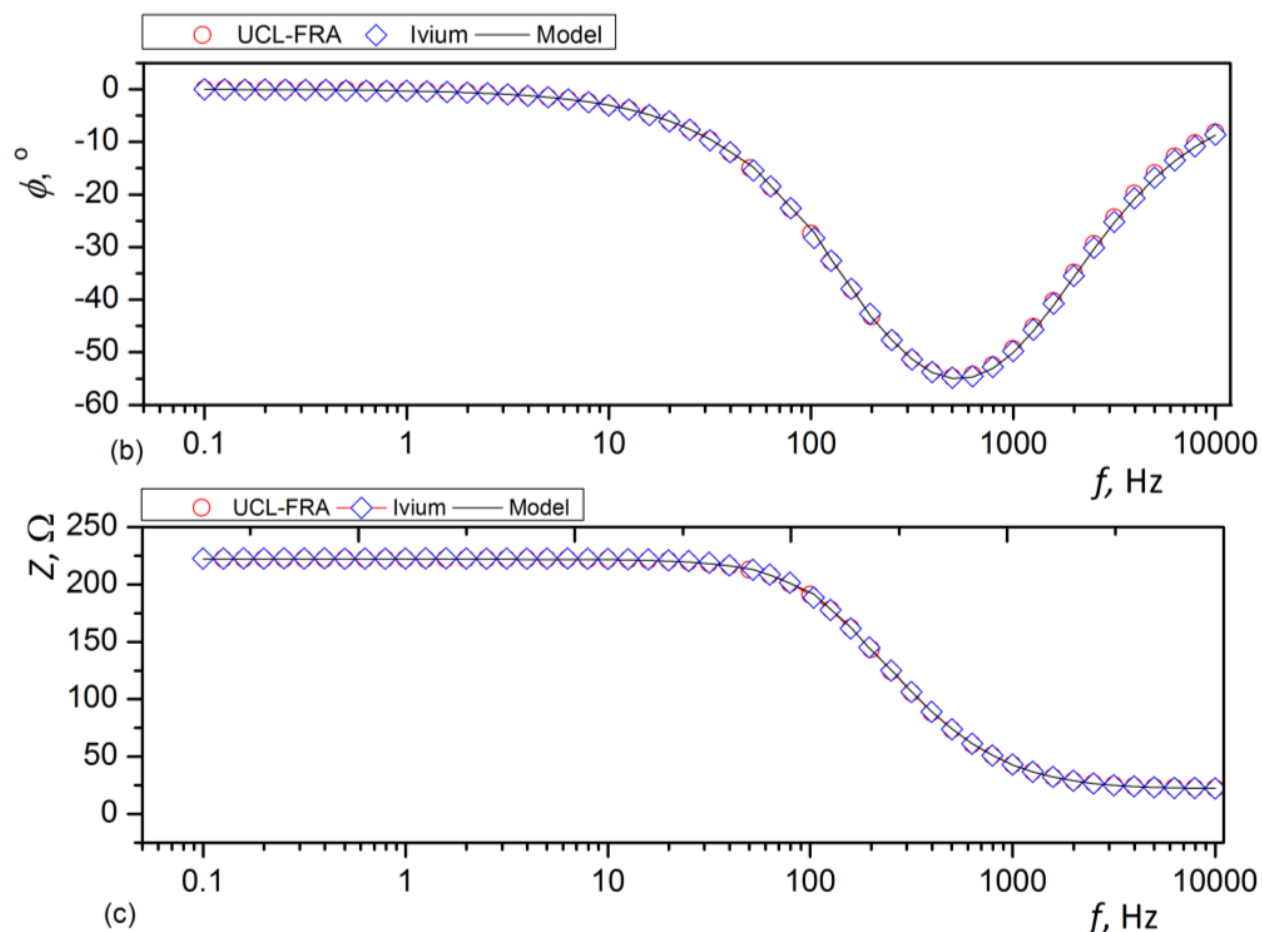

Figure 4. (a) Nyquist and (b-c) Bode plots for a single response channel using UCL-FRA and commercial Ivium systems compared with the modeled transfer function. Frequency sweep between $10 \mathrm{kHz}$ and $0.1 \mathrm{~Hz}, A C$ amplitude $0.1 \mathrm{~V}$ for a DC signal of $2 \mathrm{~V}$ with 10 frequencies per decade.

\section{Electrical impedance in multi-channel mode}

Operation in multi-channel mode imposes more of a challenge on the DAQ system. Lower cost DAQ hardware uses a single clock that triggers each of the channels sequentially. A phase shift between each of the channels is therefore possible at high frequencies and care should be taken when introducing additional channels to the measurement. Higher end hardware, with dedicated clocks on each channel, avoids this potential artefact. Figure 5 shows the response from 5 channels on the DAQ system (Z1 - Z5) connected to the circuit described in Figure 2(c). In the Nyquist projection the system shows little difference between each channel across the full frequency range, so allowing an accurate measurement of the real resistances in the circuit. In the Bode projection it can be seen that some phase difference is introduced at high frequency. However, below $3 \mathrm{kHz}$ this difference is less than 3 degrees.

Overall, these results suggest that this DAQ/LabVIEW interface system can be used for multichannel EIS data acquisition over the frequency range of $3 \mathrm{kHz}$ to $0.1 \mathrm{~Hz}$. 

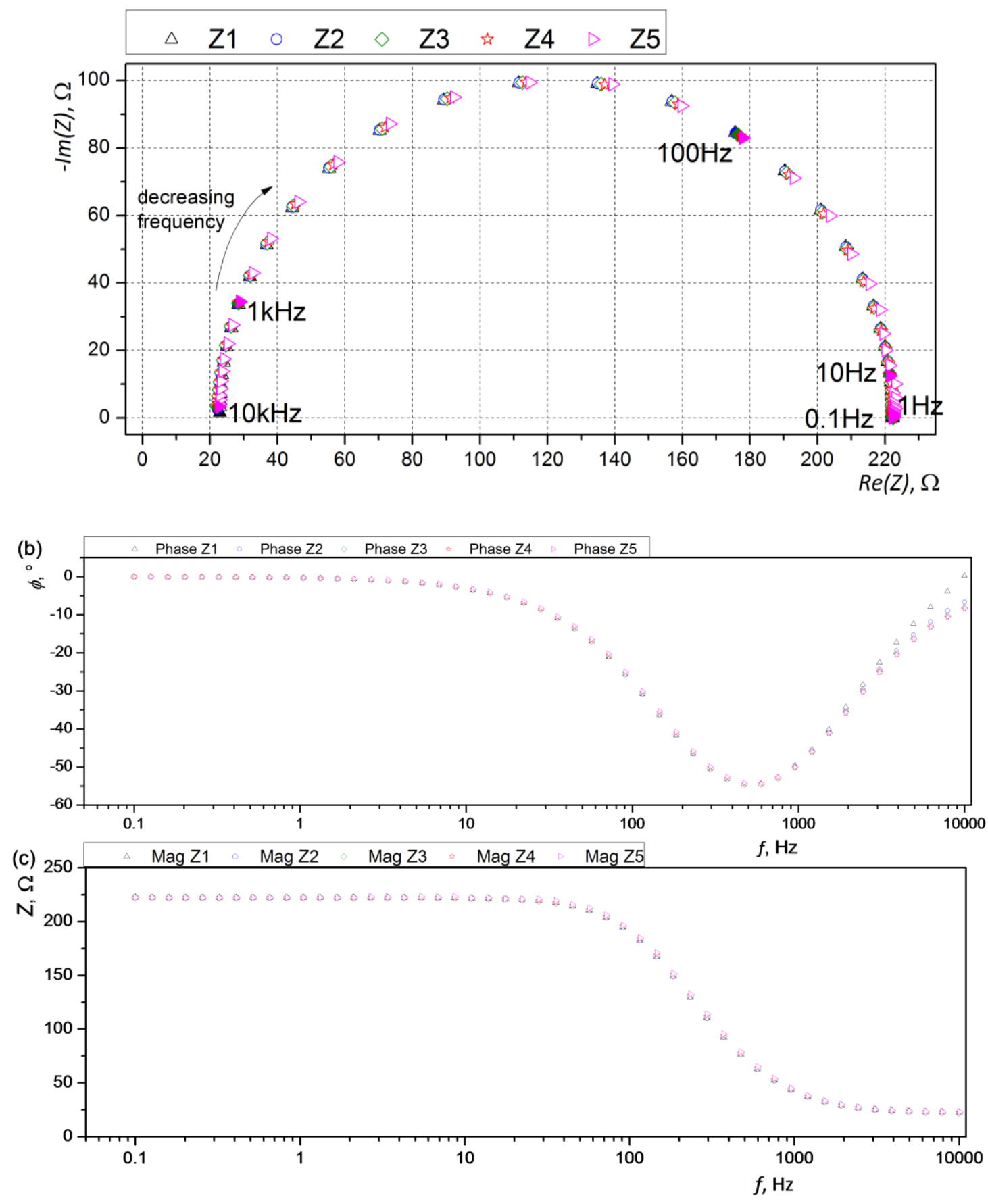

Figure 5. (a) Nyquist plots and (b-c) Bode plots showing the response from 5 channels, sampled concurrently, using the circuit described in Figure 2(c) with UCL-FRA. Frequency sweep between $10 \mathrm{kHz}$ and $0.1 \mathrm{~Hz}, A C$ amplitude $0.1 \mathrm{~V}$ for a DC signal of $2 \mathrm{~V}$ with 10 frequencies per decade.

\section{Electrochemical impedance spectroscopy on power sources}

Power sources are typically very low impedance devices and notoriously challenging for FRAs. A lead acid battery was tested (in discharge) to assess the performance of the system in active mode when incorporating an electronic load. Figure 6 shows the Nyquist and Bode (phase and magnitude) responses for the UCL and commercial (Ivium) systems. Discharge from the battery was performed in galvanostatic mode at $1.5 \mathrm{~A}$ using the set-up of Figure 1 (b). The measurement was achieved with a lowest frequency of $1 \mathrm{~Hz}$, to avoid discharging the battery too much due to the longer measurement time at low frequencies. 
The Nyquist plot shows that there is a difference (shift) in the real impedance between the DAQ/LabVIEW system and that from the Ivium analyser. However, the form of the Nyquist plot is very similar for each, exhibiting the shape of two depressed semi-circular arcs, representing two processes with different time constants (RC combinations). This is typical of an EIS response for a battery of this kind [14]. The systematic difference in real resistance between the two $(<10 \mathrm{~m} \Omega)$ is attributed to the different leads and connectors used for each system causing different ohmic resistance.

The Bode plots in Figure 6(b-c) show that the two magnitudes are very similar across the range, although the one recorded with the UCL-FRA exhibits a constant lower resistance associated with the different lead configuration. The two phases remain in close agreement up to a frequency of $\sim 3 \mathrm{kHz}$. Above this frequency, the bandwidth limitation of the load starts to become apparent.

(a)

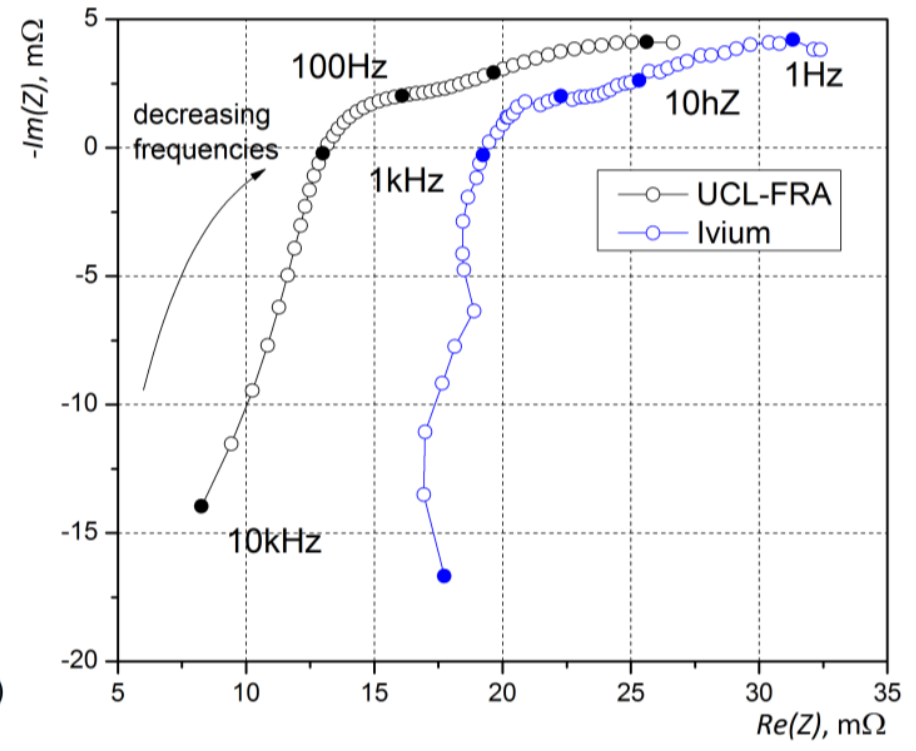

(b)
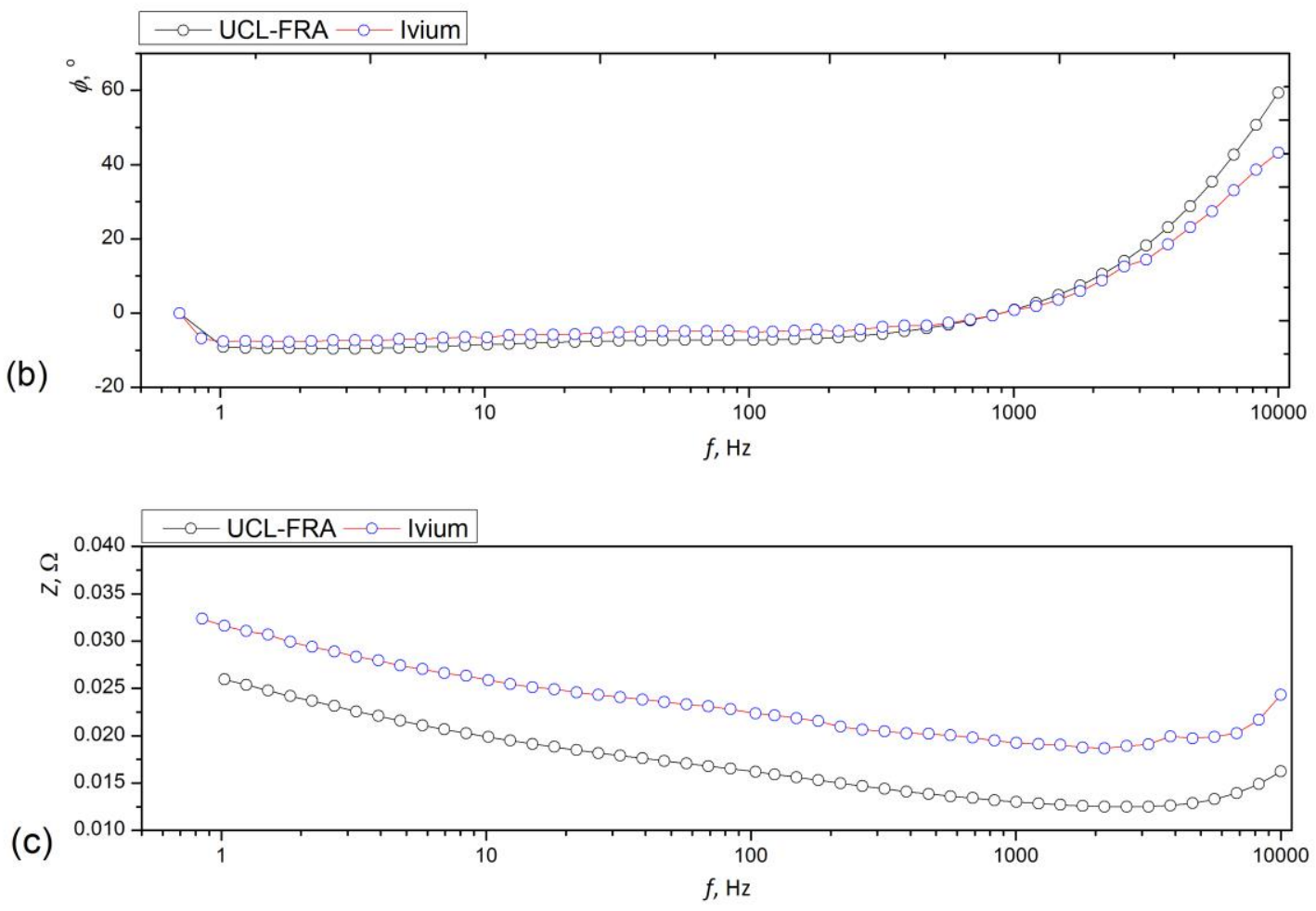

Figure 6. Electrochemical impedance response for UCL-FRA and commercial FRA (Ivium) in the form of: (a) Nyquist, and Bode, (b) phase and (c) magnitude for a lead acid battery being discharged at 1.5 A. Frequency sweep between $10 \mathrm{kHz}$ and $1 \mathrm{~Hz}, \mathrm{AC}$ amplitude $75 \mathrm{~mA}$ for a DC signal of $1.5 \mathrm{~A}$ with 10 frequencies per decade. 


\section{Conclusion}

A DAQ/LabVIEW interface has been developed capable of performing frequency response analysis for electrochemical impedance spectroscopy of low-impedance power sources. The system was tested using electrical circuits to demonstrate that multi-channel data acquisition is possible (here using 5 channels) without significant phase shift between the channels up to $3 \mathrm{kHz}$.

EIS on a battery discharging at $1.5 \mathrm{~A}$ compared favorably with a commercial FRA; the main difference being a systematic shift in real impedance and high frequency inductance, both of with can be attributed to differences in the cabling used for each arrangement.

This method is attractive for applications where a low cost solution is required for measuring electrochemical power systems and a multichannel input is needed. For example, performing individual cell EIS on fuel cell stacks or on-board diagnostics.

Acknowledgements: The authors would like to acknowledge University College London and Intelligent Energy Ltd. for supporting the work of Meyer, the EPSRC Supergen Fuel Cells programme (EP/G030995/1) and EPSRC Flexible Fuel Cell project (EP/G04483X/1) for supporting Brett's research.

\section{References}

[1] V. Sense, M. Cell, Scribner Associate-Tutorial, 1-5.

[2] M. E. Orazem, B. Tribollet, Electrochemical Impedance Spectroscopy, Wiley, Gainsville, USA 2008, p. 91-95.

[3] J.-P. Diard, B. Le Gorrec, C. Montella, Cinetique electrochimique. Hermann, 1996, p. 231241.

[4] F. L. Floyd, S. Avudaiappan, J, Gibson, Prog. Org. Coat. 66 (2009) 8-34

[5] S. Rodrigues, N. Munichandraiah, A. K. Shukla, J. Power Sources 87 (2000) 12-20

[6] E. Karden, S. Buller, R. W. D. Doncker, J. Power Sources 85 (2000) 72-78

[7] X. Yuan, H. Wang, J. Colinsun, J. Zhang, Int. J. Hydrogen Energ. 32 (2007) 4365-4380

[8] Q. Huang, R. Hui, B. Wang, J. Zhang, Electrochim. Acta 52 (2007) 8144-8164.

[9] X.-Z. Yuan, Electrochemical Impedance Spectroscopy in PEM fuel Cells, Springer 2010, p 193313.

[10] I. A. Schneider, H. Kuhn, A. Wokaun, G. G. Scherer, J. Electrochem. Soc. 152 (2005) A2092A2103.

[11] S. J. Andreasen, J. L. Jespersen, and S. Kaer, Fuel Cells 4 (2009) 463-473

[12] C. E. Shannon, Proceedings of IEEE 86 (1998) 447-457.

[13] Agilent Technologies, Agilent Sales and Support Office, 5951-2826 (2004) 31.

[14] H. Blanke, O. Bohlen, S.Buller, J. Power Sources, 144 (2005) 418-425

(C) 2013 by the authors; licensee IAPC, Zagreb, Croatia. This article is an open-access article distributed under the terms and conditions of the Creative Commons Attribution license (http://creativecommons.org/licenses/by/3.0/) 